\title{
TOPOLOGICAL SEMICONJUGACY OF PIECEWISE MONOTONE MAPS OF THE INTERVAL
}

\author{
BY
}

BILL BYERS

\begin{abstract}
This paper establishes a topological semiconjugacy between two piecewise monotone maps of the interval which have the same kneading sequences and do not map one turning point into another, whenever itineraries under the second map are given uniquely by their invariant coordinate. Various examples are given and consequences obtained.
\end{abstract}

0. Introduction. In recent years there has been a great deal of interest in understanding the complicated asymptotic behaviour which arises in studying the iteration of maps of the interval [1, 3-5, 7, 10]. In particular, Guckenheimer [4] classifies maps with negative Schwarzian derivative and one critical point up to topological equivalence by the systematic use of the Milnor-Thurston kneading theory.

In this paper we apply this kneading theory to piecewise monotone, continuous maps and show that within a very general class of maps with the same kneading sequences those maps $\tau$ for which the invariant coordinate is injective serve as models in the sense that there is a surjective, semiconjugacy from any map onto $\tau$ (Theorem 5). It follows (Proposition 7) that any two of these maps (with injective invariant coordinates and equivalent kneading sequences) are topologically equivalent. In particular any two expanding maps with equivalent kneading sequences are topologically conjugate. This result implies that (Proposition 8): A map with one turning point and a kneading sequence which is not eventually periodic is topologically semiconjugate to a map $\tau_{\mu}$ of the form $\tau_{\mu}(x)=\mu x(1-x)$.

1. Preliminaries. All maps $\tau:[0,1] \rightarrow[0,1]$ will be continuous and piecewise monotone, i.e. there exists a minimal partition $0=C_{0}<C_{1}<\cdots<C_{m-1}<C_{m}=1$ of $[0,1]$ such that $\tau \mid\left[C_{i-1}, C_{i}\right]$ is strictly increasing or strictly decreasing.

For such maps the kneading theory of Milnor and Thurston [5] applies. Let $I_{1}=$ $\left[C_{0}, C_{1}\right), I_{m}=\left(C_{m-1}, C_{m}\right]$ and $I_{k}=\left(C_{k-1}, C_{k}\right), k=2, \ldots, m-1$. Then, using the notation of [4], associated to each point $x \in[0,1]$ is a sequence $\underline{A}(x)=\left\{A_{n}(x)\right\}_{n=0}^{\infty}$, called the itinerary of $x$, where $A_{n}(x)=I_{k}$ or $C_{k}$ according as $\tau^{n}(x) \in I_{k}$ or $\tau^{n}(x)=C_{k}$. The points ' $C_{k}$ ' are called turning points for $\tau$ and their itineraries are called the kneading sequences of $\tau$. An orientation-preserving topological equivalence between maps $\tau_{1}$ and $\tau_{2}$ must preserve kneading sequences.

Received by the editors October 8, 1980 and, in revised form, May 271981.

1980 Mathematics Subject Classification. Primary 54H20; Secondaty 58F99.

() 1983 American Mathematical Society 0002-9947/82/0000-0759/\$09.00 
Define $\varepsilon\left(I_{k}\right)=+1$ if $\tau \mid I_{k}$ is increasing, $\varepsilon\left(I_{k}\right)=-1$ if $\tau \mid I_{k}$ is decreasing and $\varepsilon\left(C_{k}\right)=$ 0 . Then given a sequence $\underline{a}=\left\{a_{n}\right\}_{n=0}^{\infty}$ of symbols $I_{k}$ and $C_{k}$, the invariant coordinate $\theta(\underline{a})=\left\{\theta_{n}(\underline{a})\right\}_{n=0}^{\infty}$ is defined by

$$
\theta_{n}(\underline{a})=\varepsilon\left(a_{0}\right) \varepsilon\left(a_{1}\right) \cdots \varepsilon\left(a_{n-1}\right) a_{n}, \quad \theta_{0}(a)=a_{0} .
$$

If $x \in[0,1]$, write $\theta(x)=\theta(\underline{A}(x))$.

If we order the symbols by

$$
-I_{n}<-C_{n-1}<-I_{n-1}<\cdots<-I_{0}<\left\{\begin{array}{l}
0 \cdot C_{k} \\
0 \cdot I_{k}
\end{array}\right\}<I_{0}<C_{1}<\cdots<I_{1}
$$

and the sequences $\theta(\underline{a})$ by $\theta(\underline{a})<\theta(\underline{b})$ if $\underline{a} \neq \underline{b}$ and $\theta_{n}(\underline{a})<\theta_{n}(\underline{b})$ for the smallest $n$ for which $a_{n} \neq b_{n}$, we then have

LEMMA 1 [5]. $x_{1}<x_{2}$ implies that $\theta\left(x_{1}\right) \leq \theta\left(x_{2}\right)$.

2. Construction of the semiconjugacy. In the following we shall always be considering pairs of continuous, piecewise monotone maps $\tau_{1}, \tau_{2}$ with turning points $C_{k}^{1}$ and $C_{k}^{2}, k=1, \ldots, m-1$, respectively, which are simultaneously increasing or decreasing on the interval $\left[0, C_{1}^{i}\right], i=1,2$. Let $I_{k}^{i}=\left(C_{k-1}^{i}, C_{k}^{i}\right), i=1,2 ; k=1, \ldots, m$. To avoid confusion we shall denote the itinerary of a point $x$ under $\tau_{1}$ by $\underline{A}(x)=$ $\left\{A_{n}(x)\right\}_{n=0}^{\infty}$ and of a point $y$ under $\tau_{2}$ by $\underline{B}(y)=\left\{B_{n}(y)\right\}_{n=0}^{\infty}$ but we shall compare $\theta(\underline{A}(x))$ with $\theta(\underline{B}(y))$ by identifying $I_{k}^{1}$ with $I_{k}^{2}$ and $C_{k}^{1}$ with $C_{k}^{2}$.

Given $x \in[0,1]$. Let $L(x)=\{y \mid \theta(y)=\theta(\underline{B}(y)) \leq \theta(\underline{A}(x))=\theta(x)\}$ and $U(x)=$ $\{y \mid \theta(y) \geq \theta(x)\}$. Suppose $\tau_{2}$ has the property that $\theta\left(y_{1}\right)=\theta\left(y_{2}\right)$ only if $y_{1}=y_{2}$ (we shall say that $\theta(y)$ is one-to-one for $\left.\tau_{2}\right)$. Then $L(x)$ intersects $U(x)$ in at most one point. If $L(x)$ and $U(x)$ are nonempty and disjoint then Lemma 1 implies that $y_{1} \leq y_{2}$ for any $y_{1} \in L(x)$ and $y_{2} \in U(x)$. Thus there exists a unique point $y=\sup L(x)=$ inf $U(x)$. We define $y=\psi(x)$ in this case and $\psi(x)=L(x) \cap U(x)$ in the case where the intersection is nonempty. Thus $\phi(x)$ is well defined whenever both $L(x)$ and $U(x)$ are nonempty.

We collect the following observations in:

Lemma 2. (a) $\psi(x)$ is defined $\Leftrightarrow \theta(\underline{B}(0)) \leq \theta(\underline{A}(x)) \leq \theta(\underline{B}(1))$.

(b) If $x \in I_{k}^{1}$ and $\phi(x)$ is defined then $\phi(x) \in \overline{I_{k}^{2}}$.

(c) $\phi\left(C_{k}^{1}\right)=C_{k}^{2}, k=1, \ldots, m$.

(d) $\phi^{-1}\left(I_{k}^{2}\right) \subseteq I_{k}^{1}, k=1, \ldots, m$.

Proof. (a) $L(x)$ and $U(x)$ are nonempty if and only if

$$
\theta(\underline{B}(0)) \leq \theta(\underline{A}(x)) \leq \theta(\underline{B}(1)) .
$$

(b) If $\theta(y) \leq \theta(x)$ then $B_{0}(y) \leq A_{0}(x)=I_{k}$. Thus $L(x) \subseteq\left[0, C_{k}^{2}\right)$ and $\phi(x) \leq C_{k}^{2}$. Similarly $U(x) \subseteq\left(C_{k-1}^{2}, 1\right]$ and $\phi(x) \geq C_{k-1}^{2}$. Thus $\phi(x) \in\left[C_{k-1}^{2}, C_{k}^{2}\right]$.

(c) $\phi\left(C_{k}^{1}\right)=\sup \left\{y \mid \theta(y) \leq \theta\left(C_{k}^{1}\right)\right\}=\sup \left\{y \mid \theta(y) \leq \theta\left(C_{k}^{2}\right)\right\}=C_{k}^{2}$.

(d) Suppose $\psi(x) \in I_{k}^{2}$. If $x=C_{j}^{1}$ then $\psi(x)=C_{j}^{2}$ by (c). Thus $x \in I_{j}^{1}$ for some $j$. Then $\phi(x) \in I_{j}^{2}$ by (a) and therefore $j=k$. 
Let $X=\{x \mid \theta(\underline{B}(0)) \leq \theta(\underline{A}(x)) \leq \theta(\underline{B}(1))\}$, the domain of definition of $\phi$. Since $\theta$ is nondecreasing for $\tau_{1}, X$ is an interval. We proceed to show that $\phi$ conjugates $\tau_{1}$ and $\tau_{2}$ and is continuous whenever $\tau_{2}^{n}\left(C_{i}^{2}\right) \neq C_{j}^{2}$ for any $i, j, n$.

Proposition 3. $\psi$ is a nondecreasing function on $X$. Moreover if $x, \tau_{1}(x) \in X$ then $\psi \tau_{1}(x)=\tau_{2} \psi(x)$ if $\tau_{1}$ and $\tau_{2}$ have the same kneading sequences.

Proof. If $x_{1}<x_{2}$ then $\theta\left(x_{1}\right) \leq \theta\left(x_{2}\right)$ and so $L\left(x_{1}\right) \subseteq L\left(x_{2}\right)$. Thus $\psi\left(x_{1}\right)=$ $\sup L\left(x_{1}\right) \leq \sup L\left(x_{2}\right)=\phi\left(x_{2}\right)$.

Now fix $x \in X$ such that $\tau_{1}(x) \in X$. If there exists a point $y$ such that $\theta(\underline{A}(x))=$ $\theta(\underline{B}(y))$ then $y=\psi(x)$ and either

(a) $A_{i}(x)$ is an interval $i=0,1, \ldots$ and $\tau_{1}^{i}(x) \in I_{k}^{1} \Leftrightarrow \tau_{2}^{i}(y) \in I_{k}^{2}, i=0,1, \ldots$, or

(b) $A_{i}(x)$ is an interval for $i=0,1, \ldots, n-1$ and $\tau_{1}^{i}(x) \in I_{k}^{1} \Leftrightarrow \tau_{2}^{i}(y) \in I_{k}^{2}, i=0$, $1, \ldots, n-1$, and $\tau_{1}^{n}(x)=C_{k}^{1}, \tau_{2}^{n}(y)=C_{k}^{2}$. In either case $\theta\left(\tau_{1} x\right)=\theta\left(\tau_{2} y\right)$ or $\psi\left(\tau_{1} x\right)=$ $\tau_{2} \psi(x)$ unless $n=0$ in (b), i.e. $y=C_{k}^{2}$ and $x=C_{k}^{1}$ in which case $\theta\left(\tau_{1} C_{k}^{1}\right)=\theta\left(\tau_{2} C_{k}^{2}\right)$ if the kneading sequence of $C_{k}^{1}$ under $\tau_{1}$ and of $C_{k}^{2}$ under $\tau_{2}$ are the same.

We now look at points $x \in X$ for which $\theta(y) \neq \theta(x)$ for all $y \in[0,1]$. Thus $y \in L(x)$ $\Leftrightarrow \theta(y)<\theta(x)$ and $y \in U(x) \Leftrightarrow \theta(y)>\theta(x)$.

For any point $z, \theta_{n}(\underline{A}(z))=\varepsilon\left(A_{0}(z)\right) \cdots \varepsilon\left(A_{n-1}(z)\right) A_{n}(z)$ and $\theta_{n-1}\left(A\left(\tau_{1} z\right)\right)=\varepsilon\left(A_{1}(z)\right)$ $\cdots \varepsilon\left(A_{n-1}(z)\right) A_{n}(z)$. Thus $\theta_{n}(z)=\varepsilon\left(A_{0}(z)\right) \theta_{n-1}\left(\tau_{1} z\right)$ and similarly $\theta_{n}(B(w))=\varepsilon\left(B_{0}(w)\right)$ $\theta_{n-1}\left(\tau_{2} w\right)$. Thus $\theta_{n}(w)$ is less than (greater than) $\theta_{n}(z) \Leftrightarrow \varepsilon\left(B_{0}(w)\right) \theta_{n-1}(w)$ is less than (greater than) $\varepsilon\left(A_{0}(z)\right) \theta_{n-1}(z)$ if $n>0$.

Suppose that $x \in I_{k}^{1}$ and $\psi(x) \in I_{k}^{2}$ where $\tau \mid\left[C_{k-1}^{1}, C_{k}^{1}\right]$ is increasing. Then $\varepsilon\left(A_{0}(x)\right)=$ +1 . $\psi(x)=\sup \left\{y \in I_{k}^{2} \mid \theta(y)<\theta(x)\right\}$ and since $\tau_{2} \mid I_{k}^{2}$ is also increasing $\tau_{2} \psi(x)=$ $\sup \left\{\tau_{2}(y) \mid y \in I_{k}^{2}\right.$ and $\left.\theta(y)<\theta(x)\right\}$. For such a $y$, if $n$ is the smallest integer for which $\theta_{n}(y)<\theta_{n}(x)$ and $n>0$ then by the above $\theta_{n-1}\left(\tau_{2} y\right)<\theta_{n-1}\left(\tau_{1} x\right)$ since $\varepsilon\left(A_{0}(x)\right)$ $=+1=\varepsilon\left(B_{0}(y)\right)$. Note that $n$ must be greater than 0 since $B_{0}(y)=A_{0}(x)$. Thus $\tau_{2}\left[L(x) \cap I_{k}^{2}\right] \subseteq L\left(\tau_{1} x\right)$ and $\tau_{2} \psi(x) \leq \psi\left(\tau_{1} x\right)$. Similarly $\tau_{2}\left[U(x) \cap I_{k}^{2}\right] \subseteq U\left(\tau_{1} x\right)$ and $\tau_{2} \psi(x) \geq \psi\left(\tau_{1} x\right)$. Thus $\tau_{2}(\psi(x))=\psi\left(\tau_{1} x\right)$.

If $x \in I_{k}^{1}, \phi(x) \in I_{k}^{2}$, and $\tau_{1} \mid\left[C_{k-1}^{1}, C_{k-1}^{2}\right]$ is decreasing then $\varepsilon\left(A_{0}(x)\right)=-1$ and

$$
\begin{aligned}
\tau_{2} \psi(x) & =\tau_{2}\left(\sup \left\{y \in I_{k}^{2} \mid \theta(y)<\theta(x)\right\}\right) \\
& =\inf \left\{\tau_{2} y \mid y \in I_{k}^{2} \text { and } \theta(y)<\theta(x)\right\}
\end{aligned}
$$

but for such a $y \theta_{n}(y)<\theta_{n}(x) \Rightarrow \theta_{n-1}\left(\tau_{2} y\right)>\theta_{n-1}\left(\tau_{1} x\right)$ and $\tau_{2}(y) \in U\left(\tau_{1} x\right)$. Thus $\tau_{2} \psi(x) \geq \phi \tau_{1}(x)$. Considering $\phi(x)=\inf \left\{y \in I_{k}^{2} \mid \theta(y)>\theta(x)\right\}$ we get $\tau_{2} \psi(x) \leq \psi\left(\tau_{1}(x)\right)$, which completes the proof for such values of $x$.

Since $\phi\left(C_{k}^{1}\right)=C_{k}^{2}$ and $\theta\left(C_{k}^{1}\right)=\theta\left(C_{k}^{2}\right)$ it remains to consider points for which $\phi(x)=$ $C_{k}^{2}$ and $x \neq C_{k}^{1}$. For such points if $y \neq C_{k}^{2}$ then $\theta(y)<($ or $>) \theta(x)$ if and only if $y<$ (or $>$ ) $C_{k}^{2}$.

Suppose $\tau_{1}$ is decreasing on [ $\left.C_{k-1}^{1}, C_{k}^{1}\right]$ and increasing on [ $\left.C_{k}^{1}, C_{k+1}^{1}\right]$. If $x \in I_{k}^{1}$ (or $\left.x \in I_{k+1}^{1}\right)$ then $\tau_{1} x>\tau_{1} C_{k}^{1}$ and $\psi\left(\tau_{1} x\right) \geq \psi\left(\tau_{1} C_{k}^{1}\right)=\tau_{2} \psi\left(C_{k}^{1}\right)=\tau_{2} \psi(x)$. On the other hand $\psi(x)=\sup I_{k}^{2}=\inf I_{k+1}^{2}$ and so $\tau_{2} \psi(x)=\inf \tau_{2} I_{k}^{2}=\inf \tau_{2} I_{k+1}^{2}$. However if $y \in I_{k}^{2}\left(y \in I_{k+1}^{2}\right)$ then $\theta(y)<\theta(x)(\theta(y)>\theta(x))$ so that $\theta\left(\tau_{2} y\right)>\theta\left(\tau_{1} x\right)$ if either $x \in I_{k}^{1}$ and $y \in I_{k}^{2}$ or $x \in I_{k+1}^{1}$ and $y \in I_{k+1}^{2}$. Thus $\tau_{2}\left(I_{k}^{2}\right) \subseteq U\left(\tau_{1} x\right)$ if $x \in I_{k}^{1}$ and $\tau_{2}\left(I_{k+1}^{2}\right) \subseteq U\left(\tau_{1} x\right)$ 
if $x \in I_{k+1}^{1}$. This implies that $\tau_{2} \psi(x) \geq \inf U\left(\tau_{1} x\right)=\left(\tau_{1} x\right)$ in both cases and so $\tau_{2} \psi(x)$ $=\psi \tau_{1}(x)$. This same reasoning applies if $\tau_{1}$ is increasing on $\left[C_{k-1}^{1}, C_{k}^{1}\right]$ which completes the proof of the proposition.

We use Proposition 3 to show

Proposition 4. If $\tau_{1}, \tau_{2}$ satisfy the conditions of Proposition 3, and $\tau_{2}(0)=0$ and $\tau_{2}(1)=0$ (or 1) in the case where we have an odd (or even) number of turning points. Suppose $\tau_{2}^{n}\left(C_{k}^{2}\right) \neq C_{l}^{2}$ for any $n>0 ; k, l=1, \ldots, m$. Then $\phi:[0,1] \rightarrow[0,1]$ is continuous.

Proof. $\psi(x)$ is defined for all $x$ since $\theta(\underline{B}(0)) \leq \theta(\underline{A}(0)) \leq \theta(\underline{A}(x)) \leq \theta(\underline{A}(1)) \leq$ $\theta(\underline{B}(1))$. Note that for points ' $x$ ' for which $\tau_{1}^{n}(x) \neq C_{k}^{1}$ and $\tau_{2}^{n}(\phi(x)) \neq C_{k}^{2}$ for any $n=0,1,2, \ldots$ and $k=1, \ldots, m$ we have $\tau_{1}^{n}(x) \in I_{k}^{1}$ if and only if $\tau_{2}^{n}(\psi(x)) \in I_{k}^{2}$. (This follows from Lemma 2.) We begin by considering such points $x_{0}$.

Let $y_{0}=\psi\left(x_{0}\right)$. Then $\bigcap_{n=0}^{\infty} \tau_{2}^{-n}\left(B_{n}\left(y_{0}\right)\right)=\left\{y_{0}\right\}$ since $\theta(y)$ is one-to-one for $\tau_{2}$. Lemma 2 and the above comment imply that $\phi\left(A_{n}\left(x_{0}\right)\right) \subseteq \overline{B_{n}\left(y_{0}\right)}$. Therefore

$$
\psi\left(\bigcap_{n=0}^{\infty} \tau_{1}^{-n}\left(A_{n}\left(x_{0}\right)\right)\right) \subseteq \bigcap_{n=0}^{\infty} \tau_{2}^{-n} \overline{B_{n}\left(y_{0}\right)}
$$

since $\tau_{2}^{n} \psi(x)=\psi \tau_{1}^{n}(x) \in \overline{B_{n}\left(y_{0}\right)}$ if $\tau_{1}^{n}(x) \in A_{n}\left(x_{0}\right)$. Let $D_{N}=\bigcap_{n=0}^{N} \tau_{2}^{-n} \overline{B_{n}\left(y_{0}\right)}$ and set $D=\bigcap_{N=0}^{\infty} D_{N}$. The $D_{N}$ form a decreasing nested sequence of closed sets with intersection $D$. Suppose $D=\left\{y_{0}\right\}$. Then if $x_{m} \rightarrow x_{0}$, we have $x_{m} \in \bigcap_{n=0}^{N} \tau_{1}^{-n} A_{n}\left(x_{0}\right)$ and so $\phi\left(x_{m}\right) \in D_{N}$ for large $m$. Thus $\phi\left(x_{m}\right) \rightarrow y_{0}$. It is not difficult to show that $D$ is an interval. However if $z \in D, z \neq y_{0}$, then $\tau_{2}^{n}(z) \in \overline{B_{n}\left(y_{0}\right)}$ and $\tau_{2}^{n}(z) \notin B_{n}\left(y_{0}\right)$ for some $n$, i.e. $\tau_{2}^{n}(z)=C_{k}^{2}$ for some $k$. Since $\tau_{2}$ is at most $m$ to 1 the set of such points is countable and so $D=\left\{y_{0}\right\}$.

We next consider points $x_{0}$ for which $\psi\left(x_{0}\right)=C_{k}^{2}$ for some $k$. Then $x_{0} \in\left(C_{k-1}^{1}\right.$, $\left.C_{k+1}^{1}\right)$. Let $x_{n} \rightarrow x_{0}$ where $x_{n} \in\left(C_{k-1}^{1}, C_{k+1}^{1}\right)$ and $\psi\left(x_{n}\right) \in\left[C_{k-1}^{2}, C_{k+1}^{2}\right]$. Then $\tau_{1} x_{n} \rightarrow \tau_{1} x_{0}$. But $\tau_{2}^{k}\left(\psi\left(\tau_{1} x_{0}\right)\right) \neq C_{j}^{2}$ and $\tau_{1}^{k}\left(\tau_{1} x_{0}\right) \neq C_{j}^{1}$ for any $j$ since in either case we would have $\tau_{2}^{k+1}\left(C_{k}^{2}\right)=C_{j}^{2}$ contrary to assumption. Thus $\psi$ is continuous at $\tau_{1} x_{0}$ and $\phi \circ \tau_{1}\left(x_{n}\right) \rightarrow$ $\phi \circ \tau_{1}\left(x_{0}\right)$ i.e. $\tau_{2} \circ \psi\left(x_{n}\right) \rightarrow \tau_{2} \circ \psi\left(x_{0}\right)$. If $\phi\left(x_{n_{k}}\right) \rightarrow y$ then $\tau_{2}(y)=\tau_{2} \psi\left(x_{0}\right)$ and $y \in\left[C_{k-1}^{2}\right.$, $\left.C_{k+1}^{2}\right]$ i.e. $y \in\left[C_{k-1}^{2}, C_{k+2}^{2}\right] \cap \tau_{2}^{-1}\left(\tau_{2} C_{k}^{2}\right)=\left\{C_{k}^{2}\right\}$. Therefore $\psi\left(x_{n}\right) \rightarrow C_{k}^{2}=\psi\left(x_{0}\right)$.

Finally consider a point $x_{0}$ for which $\phi \tau_{1}^{n}\left(x_{0}\right)=\tau_{2}^{n} \psi\left(x_{0}\right)=C_{k}^{2}$ and $n>0$. Let $U$ be a neighbourhood of $\phi\left(x_{0}\right)$ small enough so that $C_{k}^{2} \notin \bigcup_{m=0}^{n=1} \tau_{2}^{k}(U)$ (such a neighbourhood exists because $\tau_{2}^{m}\left(\phi\left(x_{0}\right)\right) \neq C_{j}^{2}$ for $\left.m<n\right)$. Then $\tau_{2}^{n} \mid U$ is a homeomorphism onto an open neighbourhood of $C_{k}^{2}$. But $\psi$ is continuous at points like $\tau_{1}^{n}\left(x_{0}\right)$ for which $\phi \tau_{1}^{n}\left(x_{0}\right)$ $=C_{k}^{2}$ by the previous result. Thus $\psi^{-1}(U)=\tau_{1}^{-n} \psi^{-1} \tau_{2}^{k}(U)$ is open. This completes the proof.

What we have shown so far is summarized by

THEOREM 5. Let $\tau_{1}, \tau_{2}$ be continuous and piecewise monotone maps of the interval with the same number of turning points and equivalent kneading sequences. Suppose that the invariant coordinate $\theta(y)$ is one-to-one for itineraries of points under $\tau_{2}$, that $\tau_{2}(0)=$ $\tau_{2}(1)=0$, and that no turning point is mapped into any other under an iterate of $\tau_{2}$. Then the map $\phi$ is a continuous, semiconjugacy from $\tau_{1}$ to $\tau_{2}$ i.e. $\phi \circ \tau_{1}=\tau_{2} \circ \psi$. 
REMARK. If $\tau_{1}(0)=0=\tau_{1}(1)$ then $\phi$ is onto since in this case $\phi(0)=0$ and $\phi(1)=$ 1.

\section{Examples and applications.}

EXAmple 1. We show that the condition that $\tau_{2}^{n}\left(C_{i}^{2}\right) \neq C_{j}^{2}$ is essential. We begin with the following example of Guckenheimer [4]:

$$
\tau_{2}(x)=\left\{\begin{array}{ll}
\mu x, & 0 \leq x \leq \frac{1}{2}, \\
\mu-\mu x, & \frac{1}{2} \leq x \leq 1,
\end{array} \text { where } \mu=\frac{1+\sqrt{5}}{2}>1 .\right.
$$

We shall show in the next section that $\theta(y)$ is one-to-one for $\tau_{2}$, but

$$
\left\{\frac{1}{2}, \frac{1+\sqrt{5}}{4}, \frac{-1+\sqrt{5}}{4}\right\}
$$

is a periodic orbit so the kneading sequence of $\tau_{2}$ is $C I_{1} I_{0} C I_{1} I_{0} \ldots$ There is no point ' $x$ ' with itinerary $\underline{a}=I_{1} I_{1} I_{0} I_{1} I_{1} I_{0} \ldots$ [4].

Let $\tau_{1}$ be any $C^{1}$ map with unique turning point $C=\frac{1}{2}$ and the same kneading sequence as $\tau_{2}$ such that $\tau_{1}(0)=\tau_{1}(1)=0$. There exists [4] a point ' $y$ ' with $\underline{A}(y)=\underline{a}$. If $\psi$ were continuous then it would be surjective and so there would exist a point ' $x$ ' with $\psi(x)=y$. Suppose $\tau_{1}^{n}(x)=\frac{1}{2}$ for some $n$, then $\tau_{2}^{n}(y)=\tau_{2}^{n} \psi(x)=\psi \tau_{1}^{n}(x)=\frac{1}{2}$ which contradicts the fact that $\underline{a}$ consists entirely of intervals. Thus $\underline{A}(x)=\underline{a}$ which is impossible. Thus $\phi$ is not continuous.

EXAmple 2. We give the following simple example to illustrate Theorem 5 (cf. [1]). We modify the map $\tau_{2}(x)=4 x(1-x)$ while keeping the same kneading sequence. Define

$$
\tau_{1}(x)= \begin{cases}6 x^{2}, & 0 \leq x \leq \frac{1}{6} \\ \frac{5}{2} x-\frac{1}{4}, & \frac{1}{6} \leq x \leq \frac{1}{2} \\ 4 x(1-x), & \frac{1}{2} \leq x \leq 1\end{cases}
$$

Then $\tau_{1}(x)$ is the type of function which we have been describing for which $\theta(x)$ is clearly not one-to-one since $\tau_{1}\left(\left[0, \frac{1}{6}\right]\right)=\left[0, \frac{1}{6}\right]$. Thus $\phi\left(\left[0, \frac{1}{6}\right]\right)=0$ and if $I$ is any interval such that $\tau_{1}^{n}(I) \subseteq\left[0, \frac{1}{6}\right]$ then $\tau_{2}^{n} \psi(I)=0$ and so $\psi(I)=0$ or 1 . If

$$
\Sigma_{\tau_{1}}=[0,1]-W^{s}(0)
$$

then $\psi \mid \Sigma_{\tau_{1}}$ is onto since $\psi\left(\frac{1}{6}\right)=0$ and

$$
\psi\left(\frac{1}{2}+\frac{\sqrt{30}}{12}\right)=1
$$

where

$$
\frac{1}{6}, \frac{1}{2}+\frac{\sqrt{30}}{12} \in \Sigma_{\tau_{1}}
$$

and for $x_{1}, x_{2} \in \Sigma_{\tau_{1}}, \phi(x)=\phi(y) \Leftrightarrow \tau^{n}(x)=\tau^{n}(y)=\frac{1}{6}$ for some $n$. 
To use Theorem 5, it is necessary to identify a class of maps with the property that the invariant coordinate is injective on itineraries. In [4] it is shown that $C^{3}$ maps $\tau$ with $\tau(0)=\tau(1)=0$, a single turning point, and negative Schwarzian derivative and no contracting periodic points have this property. The same is true for maps, one of whose iterates is expanding.

Definition. A piecewise monotone map $\tau$ is expanding if there exists a constant $\lambda>1$ such that $|\tau(x)-\tau(y)| \geq \lambda|x-y|$ whenever both $x$ and $y$ lie in the same inter$\operatorname{val}\left[C_{i}, C_{i+1}\right]$.

Proposition 6. Suppose $\tau^{m}$ is expanding for some $m$. Then $\theta(x)=\theta(y)$ only if $x=y$.

Proof. If $\theta(x)=\theta(y)$ there are two possibilities:

(a) $A_{n}(x)=A_{n}(y) \neq C_{i}, n=0,1,2, \ldots$, or

(b) $A_{n}(x)=A_{n}(y) \neq C_{i}, n=0, \ldots, k-1$ and $\tau^{k}(x)=\tau^{k}(y)=C_{j}$.

In case (a) $\left|\tau^{k m}(x)-\tau^{k m}(y)\right| \geq \lambda^{k}|x-y|$ and so $\tau^{m}(x)=\tau^{m}(y)$. Thus we are in case (b) if we let $k$ be the smallest integer such that $\tau^{k}(x)=\tau^{k}(y)$. Then either $\tau^{k-1}(x)=$ $\tau^{k-1}(y)$ or $A_{k-1}(x) \neq A_{k-1}(y)$. This is impossible unless $x=y$.

In [5] Milnor-Thurston show that for any map $\tau$ with growth number $s=s(\tau)>1$, there is a semiconjugacy from $\tau$ onto a map $F_{s}$ of constant slope $s$. Thus every such map has a piecewise linear expanding "model". However the Milnor-Thurston model is not unique in the sense that there may be semiconjugacies onto different maps of constant slope, in particular the number of turning points may be reduced. If $\tau$ has a unique turning point and the turning point $C$ of $F_{s}$ satisfies $F_{s}^{n}(c) \neq c$ for $n=1,2, \ldots$ then the kneading sequences are identical [6].

A natural consequence of Theorem 5 is:

Proposition 7. If $\tau_{1}:[0,1] \rightarrow[0,1]$ are as in Theorem 5 but $\tau_{1}(0)=\tau_{1}(1)=0$ and $\theta(x)$ is one-to-one for $\tau_{1}$ then $\psi$ is a homeomorphism, i.e. $\tau_{1}$ and $\tau_{2}$ are topologically conjugate.

Proof. From Theorem 5 we have nondecreasing, onto maps $\phi_{1}, \psi_{2}$ such that $\phi_{1} \circ \tau_{1}=\tau_{2} \circ \phi_{1}$ and $\psi_{2} \circ \tau_{2}=\tau_{1} \circ \psi_{2}$. Then $\psi_{2} \circ \psi_{1} \circ \tau_{1}=\psi_{2} \circ \tau_{2} \circ \psi_{1}=\tau_{1} \circ \psi_{2} \circ \psi_{1}$ where $\psi_{2} \circ \psi_{1}$ is continuous, nondecreasing, and onto. Let $\psi=\psi_{2} \circ \psi_{1}$. Then $\phi(x)=x$ for the dense set of points for which $\tau_{2}^{n} \psi(x) \neq C_{i}^{2}$ for any $n, i$. By continuity $\psi$ is the identity. Similarly $\phi_{1} \circ \psi_{2}=$ identity and $\psi_{1}, \psi_{2}$ are homeomorphisms.

Proposition 8. If $\tau$ is any piecewise monotone map with $\tau(0)=\tau(1)=0$ and $a$ unique turning point ' $C$ ' whose kneading sequence is not eventually periodic then $\tau$ is topologically semiconjugate to a member of the series $\tau_{\mu}(x)=\mu x(1-x)$.

ProOF. $\tau$ has the kneading sequence of some $\tau_{\mu}$ [4]. Now the maps $\tau_{\mu}$ have at most one periodic attractor which (if it exists) always attracts the critical point $x=\frac{1}{2}[8,9]$. This would make the kneading sequence of $\tau_{\mu}$ eventually periodic. This is excluded and therefore $\tau_{\mu}$ has no periodic orbit and therefore $\theta(y)$ is 1-1 for $\tau_{\mu}$. The result now follows from Theorem 5. Of course if $\theta(x)$ is $1-1$ for $\tau$, then the $\tau$ is conjugate to $\tau_{\mu}$. 


\section{REFERENCES}

1 M.V. Jacobson, On smooth mappings of the circle into itself, Math. USSR-Sb. 14 (1971), 161185.

2 , Topological and metric properties of one dimensional endomorphisms, Dokl. Akad. Nauk SSSR 243 (1978), 866-869.

3. J. Guckenheimer, On the bifurcation of maps of the interval, Invent. Math. 39 (1977), 165-178.

4. - Sensitive dependence to initial conditions for one dimensional maps, Comm. Math. Phys. 70 (1979), 133-160.

5. M. Milnor and W. Thurston, On iterated maps of the interval. I, preprint, Princeton, 1977. (A complete bibliography may be found in [7].)

6. J. Milnor, A piecewise linear model for kneading, Handwritten Notes, 1976.

7. Pierre Collet and Jean-Pierre Eckmann, Iterated maps on the interval as dynamical systems, Birkhäuser, Basel, 1980.

8. P. Fatou, Sur les équations fonctionnelles, Bull. Soc. Math. France 47 (1919), 161-270; 48 (1920), 208-314.

9. G. Julia, Memoire sur l'iteration des fonctions rationelles, J. Math. Pures Appl. 4 (1918), 47-245.

10. L. Jonker, Periodic orbits and kneading invariants, Proc. London Math. Soc. 39 (1979), 428-450. (A complete bibliography may be found in [7].)

Department of Mathematics, Concordia University, Montreal, Quebec, Canada 